\title{
Cirurgia oncoplástica e reconstrutiva da mama: Reunião de Consenso da Sociedade Brasileira de Mastologia
}

\author{
Oncoplastic and reconstructive surgery of the breast: \\ Consensus Meeting of the Brazilian Society of Mastology
}

Cicero Urban', Ruffo Freitas-Junior², Gustavo Zucca-Matthes³ ${ }^{3}$, Jorge Villanova Biazús ${ }^{4}$,

Fabricio Palermo Brenelli ${ }^{5}$, Douglas de Miranda Pires ${ }^{6}$, André Vallejo da Silva ${ }^{7}$, Mauricio de Aquino Resende ${ }^{8}$,

Régis Resende Paulinelli², Clécio Ênio Murta de Lucena ${ }^{6}$, Elvis Barbosa ${ }^{9}$, Carlos Alberto Ruizio ${ }^{10}$,

Rubens Murilo de Athayde Prudêncio ${ }^{11}$, Vilmar Marques de Oliveira ${ }^{12}$, Rodrigo Villaverde ${ }^{13}$

\section{Descritores}

Neoplasias da mama

Câncer de Mama

Reconstrução da mama

Consenso

Brasil

\section{RESUMO}

Objetivo: Apesar do grande número de publicaçóes em cirurgia oncoplástica e reconstrutiva da mama, diversas questóes permanecem controversas. Assim, o objetivo desta Reuniáo de Consenso, foi desenvolver um guia prático de recomendaçóes baseadas nas melhores evidências disponíveis na literatura. Método: Os painelistas foram os membros da Comissão de Cirurgia Oncoplástica e Reconstrutiva da Sociedade Brasileira de Mastologia. A reunião foi realizada em agosto de 2015 em Bento Gonçalves (RS). Cada painelista recebeu e respondeu previamente um questionário com 46 itens, com base na melhor evidencia cientifica e em sua experiência. Foi considerado consenso a concordância de 75\% entre painelistas. Resultados: Houve consenso em 25 itens, dos quais para oito houve concordância de $100 \%$. O mais importantes foram: comprometimento das margens em cirurgia oncoplástica pode ser resolvido com ampliação de margens na maioria dos casos; tumores multifocais não são contraindicação para cirurgia oncoplástica; idade >70 anos não representa contraindicação para uso de técnicas oncoplásticas; reconstrução imediata pode ser indicada com segurança para a maioria das candidatas à mastectomia; pacientes com indicaçấo de radioterapia pós-mastectomia podem ser submetidas à reconstrução imediata, devendo ter ciência dos riscos maiores para mau resultado estético; mastectomia com preservação do complexo areolopapilar é segura nos casos de câncer; radioterapia após a mastectomia com preservação do complexo areolopapilar nâo está indicada fora dos critérios clássicos de irradiação do plastrão; tela abdominal reduz chances de hernia no caso de reconstrução com TRAM. Conclusão: através desta reunião foi possível estabelecer importantes pontos consensuais de acordo com a opiniấo dos especialistas, que poderấo auxiliar os mastologistas na tomada de decisóes em cirurgias oncoplásticas e reconstrutivas da mama.

Trabalho realizado pela Comissão de Cirurgia Oncoplástica e Reconstrutiva da Mama da Sociedade Brasileira de Mastologia - Rio de Janeiro (RJ), Brasil.

'Unidade de Mama do Hospital Nossa Senhora das Graças; Curso de Medicina e Programa de Pós graduação em Biotecnologia da Universidade Positivo - Curitiba (PR), Brasil.

${ }^{2}$ Serviço de Ginecologia e Mama do Hospital Araújo Jorge da Associação de Combate ao Câncer em Goiás - Goiânia (G0), Brasil.

${ }^{3}$ Unidade de Mama do Hospital de Câncer de Barretos - Barretos (SP), Brasil

${ }^{4}$ Hospital de Clínicas de Porto Alegre; Faculdade de Medicina da Universidade Federal do Rio Grande do Sul (UFRGS) - Porto Alegre (RS), Brasil.

${ }^{5}$ Divisão de Oncologia Mamária do Departamento de Tocoginecologia da Universidade Estadual de Campinas (UNICAMP) - Campinas (SP);

Centro Oncológico Antônio Ermínio de Moraes, Hospital Beneficência Portuguesa - São Paulo (SP), Brasil.

${ }^{6}$ Clínica de Mastologia da Santa Casa de Belo Horizonte - Belo Horizonte (MG), Brasil.

${ }^{7}$ Serviço de Mastologia da Universidade Federal Fluminense (UFF) - Rio de Janeiro (RJ), Brasil.

${ }^{8}$ Universidade Federal de Sergipe (UFS) - Aracajú (SE), Brasil.

${ }^{9}$ Hospital Geral de Fortaleza - Fortaleza (CE), Brasil.

${ }^{10} \mathrm{Clínica}$ Ginecológica do Hospital das Clínicas da Faculdade de Medicina da Universidade de São Paulo (USP) - São Paulo (SP), Brasil.

${ }^{11}$ Hospital e Maternidade São Cristóvão - São Paulo (SP), Brasil.

${ }^{12}$ Santa Casa de São Paulo - São Paulo (SP), Brasil.

${ }^{13}$ Programa de Pós-graduação em Biotecnologia da Universidade Positivo - Curitiba (PR), Brasil.

Endereço para correspondência: Cicero de Andrade Urban - Rua Angelo Domingos Durigan, 1240, casa 1 - Cascatinha - CEP: 82020 -452 -

Curitiba (PR), Brasil - Email: cicerourban@hotmail.com

Conflito de interesses: Ruffo Freitas-Junior é presidente da Sociedade Brasileira de Mastologia; Gustavo Zucca-Matthes é consultor mentor J\&J.

Recebido em: 23/10/2015. Aceito em: 25/10/2015. 


\section{Keywords}

Breast neoplasms

Breast Cancer

Breast reconstruction

Consensus

Brazil

\section{ABSTRACT}

Objective: Despite the large number of publications in oncoplastic and breast reconstructive surgery, several issues remain controversial. The aim of this Consensus Meeting was to develop a practical guide of recommendations based on the best evidence in the literature. Method: All panelists were members of the Oncoplastic Commission of the Brazilian Society of Mastology. The Consensus Meeting was held in Bento Gonçalves (RS), in August 2015. Each panelist received and answered a questionnaire with 46 items, based on the best evidence in the literature and in their expertise. It was considered consensus the agreement of 75\% between panelists. Results: There was consensus on 25 items, of which eight were for $100 \%$ agreement. The most important of these topics were: involvement of the margins in oncoplastic surgery can be solved by resection of margins in most cases; multifocal tumors is not a contraindication for oncoplastic surgery; age $>70$ years is not a contraindication for use of oncoplastic techniques; immediate reconstruction can be performed safely to most candidates for mastectomy; patients for post-mastectomy radiotherapy may be subject to immediate reconstruction and should be aware of the risks for poor aesthetic result; mastectomy with preservation of the nipple and areola complex is safe in cancer; radiotherapy after mastectomy with preservation of the nipple and areola complex is not indicated outside the classical criteria of irradiation chest wall; abdominal mash reduces chances of hernia in TRAM flaps. Conclusion: In this meeting it was possible to establish important consensus points according to the opinion of experts, which can help breast surgeons in their decision-making in oncoplastic and reconstructive surgery of the breast.

\section{Introdução}

Com o aumento da sobrevida câncer específica ${ }^{1}$ e da sobrevida global ${ }^{2}$ de mulheres com câncer de mama, a cirurgia oncoplástica e reconstrutiva passou a representar um grande avanço para essas pacientes ${ }^{3-7}$. A preocupação com a estética e com a qualidade de vida estão presentes no tratamento oncológico em todas as suas fases ${ }^{8,9}$. As mutilaçôes do passado já não se justificam mais do ponto de vista oncológico ${ }^{10} \mathrm{e}$ biológico ${ }^{11}$ para a maioria das pacientes com câncer de mama inicial, diagnosticadas na era dos exames de imagem ${ }^{12,13} \mathrm{e} \mathrm{da}$ oncoplastia ${ }^{14}$. Embora a cirurgia oncoplástica e reconstrutiva tenha tornado o tratamento cirúrgico mais individualizado e permitido melhorias importantes ${ }^{15-37}$, existem controvérsias ainda em vários aspectos no manejo das pacientes ${ }^{38,39}$. Nem todos os procedimentos reconstrutivos mamários possuem nível de evidência elevado na literatura e existem decisôes que se baseiam em critérios relacionados à experiência individual de cada cirurgiâa ${ }^{19,21,25,27,40-43}$. Soma-se a isso também a grandeza territorial do Brasil e sua diversidade epidemiológica ${ }^{44} \mathrm{e}$ de acessibilidade aos melhores métodos diagnósticos e terapêuticos ${ }^{45,46}$.

Assim, o propósito desta Reuniáo de Consenso da Sociedade Brasileira de Mastologia (SBM), realizada em Bento Gonçalves (RS) em agosto de 2015, foi o de desenvolver um guia prático de recomendaçôes baseadas nas melhores evidências disponíveis na literatura e na opiniấo dos experts para auxiliar os mastologistas nas suas decisões e nas suas condutas nas cirurgias oncoplásticas e reconstrutivas da mama. Os painelistas consideram este esforço da SBM um instrumento válido e importante para melhoria dessas cirurgias no Brasil, tanto no sistema público quanto no privado.

\section{Metodologia}

Os painelistas que participaram do Consenso são os membros da Comissão de Cirurgia Oncoplástica e Reconstrutiva da Mama da SBM (triênio 2013-2016). Todos os membros com experiência reconhecida e dedicação exclusiva à cirurgia oncoplástica e reconstrutiva da mama. $\mathrm{O}$ modelo e a metodologia utilizados neste Consenso foram adaptados daqueles existentes no St. Gallen International Expert Consensus Panel e no ASCO Guidelines $^{52-54}$. O questionário foi enviado aos painelistas com três semanas de antecedência, dando prioridade para questóes controversas, para as quais a decisão dos painelistas poderia auxiliar na clínica diária. As respostas foram realizadas individualmente, de maneira anônima e sem interferência externa ou contato entre os membros. Todos os comentários e sugestôes eram possíveis de serem inseridos nesse primeiro momento, e poderiam ser discutidos no dia da reunião pública no Simpósio de Oncoplástica no Vale dos Vinhedos em Bento Gonçalves (RS). Três respostas possíveis eram permitidas para a maioria das 46 questóes: sim/ não/abstenção. A abstenção poderia ser utilizada nos casos onde não existissem dados suficientes, ou o painelista não tivesse experiência ou conhecimento suficiente naquele tópico, ou então que tivesse conflito de interesse que o impedisse de votar. $\mathrm{O}$ consenso era atingido quando existia concordância de pelo menos $75 \%$ 
dos painelistas. As questóes que não atingiram consenso nessa primeira votação foram novamente discutidas durante a reunião plenária e colocadas para votação presencial. As seguintes áreas foram discutidas entre os painelistas:

- conceito e filosofia;

- treinamento cirúrgico;

- reconstrução pós-mastectomia;

- próteses e expansores;

- retalhos;

- reconstrução da aréola e mamilo;

- lipofilling.

\section{Resultados}

\section{Perfil dos painelistas}

Treze especialistas em Mastologia, membros da Comissão de Cirurgia Oncoplástica e Reconstrutiva da SBM para o triênio de 2013-2016, participaram desse painel, todos eles do sexo masculino. A idade dos especialistas variou de 38 a 63 anos, sendo que todos apresentam mais de 10 anos de experiência na especialidade. Dentre esses profissionais, a grande maioria $(69,3 \%)$ realiza mais de 100 cirurgias oncoplásticas e reconstrutivas por ano e $84,6 \%$ dedicam-se a dividir sua atenção profissional tanto com pacientes do Sistema Único de Saúde (SUS), quanto com atendimento privado.

\section{Cirurgia oncoplástica mamária: definição}

O painel, após muita discussão, chegou ao consenso, com 100\% de concordância para a definição do termo mais adequado como sendo: cirurgia oncoplástica e reconstrutiva da mama. Este representa melhor o conjunto de técnicas que possui a finalidade específica de melhorar os resultados oncológicos e estéticos no tratamento do câncer de mama ${ }^{16,47-51}$. Definiu-se que não seria necessária uma certificação específica dentro da Mastologia. Contudo, recomenda-se que os mastologistas interessados nesse campo cirúrgico e que não tiveram a oportunidade de realizar uma formação mais específica devem buscar treinamento em técnicas de reconstrução mamária. Para isso, existem os cursos práticos hands-on, cada qual com suas características próprias. Uma análise detalhada da SBM será realizada para que sejam definidos critérios de duração e conteúdo para cada curso a ser chancelado pela entidade. Os cursos apoiados pela SBM serão individualizados por suas condiçôes especiais e avaliados por comissôes específicas. Discutiu-se, ainda, que é papel da SBM trabalhar e fiscalizar como deve ser formado e treinado seu especialista durante a residência em Mastologia.

\section{Reconstrução parcial}

As diversas técnicas existentes são possíveis de serem aplicadas, devendo ser individualizadas, dentro de um contexto de maior personalização oncológica e estética do tratamento cirúrgico. Todos concordaram que o uso das técnicas oncoplásticas permitem uma abordagem tumoral ampla, favorecendo margens oncológicas seguras, podendo ser uma ferramenta importante para reduzir os riscos de margens comprometidas e de reoperaçôes. Com relação às margens, não houve consenso sobre a necessidade de avaliação anatomopatológica no peroperatório. Contudo, no caso de margens comprometidas no exame peroperatório, a ampliação pode ser indicada. Caso persistam margens positivas no pós-operatório (no exame anatomopatológico definitivo), a ampliação de margens ou a mastectomia deve ser indicada, a depender da individualidade de cada caso. Mulheres portadoras de assimetria mamária e gigantomastia são melhores candidatas à cirurgia oncoplástica mamária. Também foi consenso que pacientes idosas (acima de 70 anos) podem ser submetidas às cirurgias oncoplásticas e reconstrutivas da mama. É necessário individualizar a solicitação de ressonância nuclear magnética das mamas para avaliação pré-operatória nas cirurgias oncoplásticas. Recomenda-se o uso de clips metálicos para guiar o reforço radioterápico no leito cirúrgico (boost). Tumores multifocais não foram considerados contraindicação para tratamento conservador com técnicas oncoplásticas. Por outro lado, nos casos de tumores multicêntricos, a conservação mamária associada a técnicas oncoplásticas foi considerada como contraindicação relativa por $53,8 \%$ dos painelistas, mas não atingiu consenso $0^{3-7,9,16,18,19,27,30,37,38,40,41,47-49}$ (Tabela 1).

\section{Reconstrução após mastectomia}

Foi consenso que biópsias retroareolares devem ser realizadas para casos que envolvam indicaçáo de mastectomia com preservação do complexo areolopapilar. Já a reconstrução imediata para casos de carcinoma inflamatório permaneceu como contraindicação relativa para reconstrução mamária.

\section{Próteses e expansores}

As próteses anatômicas são consideradas as mais indicadas para a maioria dos casos de reconstrução mamária pós-mastectomia, contudo, tendo em vista as condiçōes e individualidades de cada paciente e a experiência do profissional, outras formas de implantes podem ser aceitas. Não houve consenso quanto ao melhor momento para troca do expansor pela prótese definitiva. Desaconselha-se o uso de implantes mamários para corrigir defeitos parciais decorrentes de cirurgias conservadoras. Estes poderão ser utilizados em situações individualizadas e alertando-se os riscos de mau resultado estético aos pacientes. A loja parcial, quando possível, deve ser considerada como primeira opção nas cirurgias reconstrutoras com próteses e expansores. Não há necessidade de substituiçâo profilática do implante a cada dez anos. Recomenda-se a substituição dos implantes nos casos de roturas, contratura capsular graus 3 e 4 
e assimetrias mamárias. Não houve consenso sobre como deve ser o acompanhamento dessas pacientes para avaliar a integridade dos implantes, com relação aos exames de seguimento a serem realizados ${ }^{14,23,24,26,28,29,31,36,39,50}$.

\section{Retalhos}

O retalho miocutâneo com o músculo reto abdominal (TRAM) é o retalho de escolha da maioria dos painelistas quando a paciente necessita de reconstrução autóloga (imediata ou tardia), principalmente o ipsilateral. A associação de tela para prevenção de hérnias é sugerida. Para pacientes submetidas à radioterapia não houve um consenso sobre a melhor opçáo entre o retalho grande dorsal ou TRAM, opçôes que dividiram o painel em $53,8 \%$ para cada tipo, tendo uma abstenção. Contudo, os retalhos autólogos são os mais indicados após radioterapia ${ }^{17,25,33,35}$.

Tabela 1. Principais aspectos que atingiram consenso pelos painelistas

Questóes aplicadas
Conceito e finalidade
A cirurgia oncoplástica e reconstrutiva da mama corresponde a um conjunto de técnicas com uma finalidade
específica que é a melhora dos resultados oncológicos e estéticos no câncer de mama
Não é necessária uma certificação específica para os Mastologistas realizarem estas cirurgias
Os painelistas entendem que é importante que exista treinamento dos mastologistas em todas as técnicas
reconstrutivas
Os cursos práticos apoiados pela SBM para aqueles que não tiveram treinamento específico são uma metodologia
adequada para auxiliar os mastologistas na sua prática

Reconstrução parcial

A maioria das pacientes com gigantomastia tem benefício com o uso de técnicas oncoplásticas

Pacientes com tumores localizados em quadrantes de risco para mau resultado estético (central, inferior e medial) têm benefício com técnicas oncoplásticas

Pacientes com assimetria importante entre as mamas se beneficiam de técnicas oncoplásticas

A maioria das pacientes candidatas à cirurgia oncoplástica não necessitam de ressonância nuclear magnética das mamas no pré-operatório

Clips no leito operatório são indicados para guiar o boost da radioterapia

$76,9 \%$

O comprometimento das margens em cirurgia oncoplástica pode ser resolvido com ampliação de margens na maioria dos casos

Tumores multifocais não são contraindicação para cirurgia oncoplástica

A idade avançada (> 70 anos) como fator isolado não representa contraindicação para o uso de técnicas oncoplásticas, nem mesmo de cirurgias bilaterais

Reconstrução após mastectomia

A reconstrução imediata pode ser indicada com segurança para a maioria das pacientes candidatas à mastectomia

A reconstrução imediata com expansores e próteses é a principal indicação nas pacientes candidatas à mastectomia

Pacientes candidatas à radioterapia pós-mastectomia podem ser submetidas à reconstruçáo imediata, desde que tenham ciência dos riscos maiores para mau resultado estético

A mastectomia com preservação do complexo areolopapilar é segura nos casos de câncer

A radioterapia após a mastectomia com preservação do complexo areolopapilar não está indicada fora dos critérios clássicos de indicação de radioterapia em plastrão

O exame intraoperatório da margem retroareolar após a mastectomia com preservação do complexo areolopapilar é recomendável

Próteses

As próteses anatômicas são as mais recomendáveis para reconstrução mamária para a maioria das pacientes

A cobertura muscular parcial (com músculo peitoral maior) é a mais recomendável para a maioria das pacientes

As próteses mamárias não necessitam de substituição preventiva após 10 anos

Retalhos autólogos

Nos casos de TRAM, as modalidades ipsilateral e monopediculado são aquelas de escolha

A tela abdominal pode reduzir as chances de hérnia e sugere-se o seu uso para maioria das pacientes

$100 \%$

Reconstrução da aréola e mamilo

O melhor momento para reconstrução do complexo areolopapilar é de 3 a 6 meses depois da cirurgia de reconstrução imediata ou tardia da mama

Dermopigmentação, retalho local ou enxerto são as técnicas melhor indicadas para a maioria das pacientes submetidas à mastectomia 


\section{Reconstrução do complexo areolopapilar}

Sugere-se que o melhor momento para realizaçáo da reconstrução do complexo areolopapilar (CAP) seria de 3 a 6 meses após a reconstrução mamária, não havendo consenso quanto à melhor técnica a ser utilizada ${ }^{21,22,34}$.

\section{Lipofilling}

O enxerto de gordura é considerado pelo painel de especialistas oncologicamente seguro após cirurgias conservadoras e mastectomias ${ }^{55-58}$.

\section{Termo de Consentimento Livre e Esclarecido}

A necessidade do uso do Termo de Consentimento Livre e Esclarecido (TCLE) em todas as cirurgias oncoplásticas e reconstrutivas da mama foi considerado consenso por todos os presentes.

\section{Agradecimentos}

A SBM agradece a participação de todos os painelistas pelas sugestôes e comentários, bem como pela revisão final desta Reunião de Consenso. Sem a participação ativa de todos os membros, não seria possível a sua realização.

\section{Patrocínio}

A SBM foi a única responsável pelo patrocínio para que todos os painelistas pudessem participar e estar presentes durante os debates que ocorreram no Vale dos Vinhedos, em Bento Gonçalves.

\section{Conflitos de interesses}

Os conflitos de interesses de todos os painelistas foram avaliados e estavam disponíveis aos painelistas durante todo o evento.

\section{Apêndice}

Membros do Painel e Respectivas Funçóes na Reunião de Consenso: Coordenadores: Cícero de Andrade Urban e Ruffo Freitas-Junior Secretário: Gustavo Zucca-Matthes

Comissão de Conflitos de Interesses: Fabrício Brenelli, Douglas Pires e Clécio Lucena

Comissão de Ética: Cícero Urban, Jorge Biazús, Régis Paulinelli. Painelistas: André Vallejo, Jorge Biazus, Maurício Resende, Vilmar Marques, Fabrício Brenelli, Regis Paulinelli, Douglas Pires, Clécio Lucena, Elvis Barbosa, Rubens Prudêncio, Carlos Ruiz. Software: Rodrigo Villaverde.

\section{Referências}

1. Allemani C, Weir HK, Carreira H, Harewood R, Spika D, Wang XS, et al. Global surveillance of cancer survival 1995-2009: analysis of individual data for $25,676,887$ patients from 279 populationbased registries in 67 countries (CONCORD-2). Lancet. 2015;385(9972):977-1010.

2. Freitas-Junior $\mathrm{R}$, Soares LR, Barrios $\mathrm{CH}$. Cancer survival: the CONCORD-2 study. Lancet. 2015;386(9992):428-9.

3. Hennigs A, Hartmann B, Rauch G, Golatta M, Tabatabai $\mathrm{P}$, Domschke C, et al. Long-term objective esthetic outcome after breast-conserving therapy. Breast Cancer Res Treat. 2015;153(2):345-51.

4. Clough KB, Kroll SS, Audretsch W. An approach to the repair of partial mastectomy defects. Plast Reconstr Surg. 1999;104(2):409-20.

5. Matthes AGZ, Rietjens M, Brenelli FP, Vieira MAC. Cirurgia oncoplástica: uma refinada alternativa para o tratamento contra o câncer de mama. Rev Soc Bras Cancerol. 2006;2:40-8.

6. Clough KB, Lewis JS, Couturaud B, Fitoussi A, Nos C, Falcou MC. Oncoplastic techniques allow extensive resections for breast-conserving therapy of breast carcinomas. Ann Surg. 2003;237(1):26-34.

7. Urban CA. Oncoplastic in a pre-paradigm era: a Brazilian perspective in an American problem. Plast Reconstr Surg.125(6):1839-41; author reply 1841-3.

8. Grabsch B, Clarke DM, Love A, McKenzie DP, Snyder RD, Bloch $S$, et al. Psychological morbidity and quality of life in women with advanced breast cancer: a cross-sectional survey. Palliat Support Care. 2006;4(1):47-56.

9. Freitas-Silva R, Conde DM, de Freitas-Júnior R, Martinez EZ. Comparison of quality of life, satisfaction with surgery and shoulder-arm morbidity in breast cancer survivors submitted to breast-conserving therapy or mastectomy followed by immediate breast reconstruction. Clinics. 2010;65(8):781-7.

10. Veronesi U, Stafyla V, Luini A, Veronesi P. Breast cancer: from "maximum tolerable" to "minimum effective" treatment. Front Oncol. 2012;2(125):1-5.

11. Fisher B. Biological research in the evolution of cancer surgery: a personal perspective. Cancer Res. 2008;68(24):10007-20.

12. Martins E, Freitas-Junior R, Curado MP, Freitas NM, De Oliveira JC, Silva CM. [Temporal evolution of breast cancer stages in a population-based cancer registry in the Brazilian central region]. Rev Bras Ginecol Obstet. 2009;31(5):219-23.

13. Mitsuyuki MC, Vieira RAC, Mauad EC, Pêra A, Mendonça MLH, Ribeiro, GHP, et al. Perfil epidemiológico de 8.380 mulheres portadoras de câncer de mama tratadas em uma única instituição ao longo de 23 anos. Rev Bras Mastol. 2009;19(S1):43.

14. Zucca-Matthes G, Manconi A, Vieira RAC, Michelli RAD, Matthes ACS. The evolution of mastectomies in the oncoplastic breast surgery era. Gland Surg. 2013;2(2):102-6.

15. McCarthy CM, Klassen AF, Cano SJ, Scott A, Vanlaeken N, Lennox PA, et al. Patient satisfaction with postmastectomy breast reconstruction: a comparison of saline and silicone implants. Cancer. 2010; 116(24):5584-91.

16. Audretsch W, Rezai M, Kolotas C, Zamboglou N, Schnabel TH, Bojar H. Onco-plastic surgery: "Target" volume reduction (BCTmastopexy), lumpectomy, reconstruction (BCT-reconstruction), and flap-supported operability in breast cancer. In: Proceedings 2nd European Congress on senology; October 2-6, 1994; Vienna, Austria; Bologna, Italy, Monduzzi, 1994:139-157. 
17. Momoh AO, Colakoglu S, Westvik TS, Curtis MS, Yueh JH, de Blacam C, et al. Analysis of areolopapilar complications and patient satisfaction in pedicled transverse rectus abdominis myocutaneous and deep inferior epigastric perforator flap breast reconstruction. Ann Plast Surg. 2012;69(1):19-23.

18. Benelli L. A new periareolar mammaplasty: the "round block" technique. Aesthetic Plast Surg. 1990;14(2):93-100.

19. Paulinelli RR, de Oliveira VM, Bagnoli F, Chade MC, Alves KL, Freitas-Junior R. Oncoplastic mammaplasty with geometric compensation: a technique for breast conservation. J Surg Oncol. 2014;110(8):912-8.

20. Lacotte B, Lejour M, Abboud M, Zielonka E. [Analysis of 156 breast reconstructions by transverse rectus abdominis muscle flap (TRAM)]. Ann Chir Plast Esthet. 1994;39(1):77-86.

21. Spear SL, Arias J. Long-term experience with nipple-areola tattooing. Ann Plast Surg. 1995;35(3):232-6.

22. Spear SL, Beckenstein MS. The nipple guard: an alternative covering for nipple-areola reconstructions with or without skin grafts. Plastic Reconstr Surg. 1997;100(6):1509-12.

23. Alderman AK, Wilkins EG, Lowery JC, Kim M, Davis JA. Determinants of patient satisfaction in postmastectomy breast reconstruction. Plast Reconstr Surg. 2000;106(4):769-76.

24. Clough KB, O’Donoghue JM, Fitoussi AD, Nos C, Falcou MC. Prospective evaluation of late cosmetic results following breast reconstruction: I. Implant reconstruction. Plast Reconstr Surg. 2001;107(7):1702-9.

25. Disa JJ, Cordeiro PG, Heerdt AH, Petrek JA, Borgen PJ, Hidalgo DA. Skin-sparing mastectomy and immediate autologous tissue reconstruction after whole-breast irradiation. Plast Reconstr Surg. 2003;111(1):118-24.

26. Spear SL, Mardini S, Ganz JC. Resource cost comparison of implant-based breast reconstruction versus TRAM flap breast reconstruction. Plast Reconstr Surg. 2003;112(1):101-5.

27. Anderson BO, Masetti R, Silverstein MJ. Oncoplastic approaches to partial mastectomy: an overview of volume-displacement techniques. Lancet Oncol. 2005;6(3):145-57.

28. Alderman AK, Kuhn LE, Lowery JC, Wilkins EG. Does patient satisfaction with breast reconstruction change over time? Two-year results of the Michigan Breast Reconstruction Outcomes Study. J Am Coll Surg. 2007;204(1):7-12.

29. Hunter JE, Malata CM. Refinements of the LeJour vertical mammaplasty skin pattern for skin-sparing mastectomy and immediate breast reconstruction. J Plast Reconstr Aesthet Surg. 2007;60(5):471-81.

30. Petit JY, De Lorenzi F, Rietjens M, Intra M, Martella S, Garusi $\mathrm{C}$, et al. Technical tricks to improve the cosmetic results of breastconserving treatment. Breast. 2007;16(1):13-6.

31. Pusic AL, Chen CM, Cano S, Klassen A, McCarthy C, Collins ED, et al. Measuring quality of life in cosmetic and reconstructive breast surgery: a systematic review of patient-reported outcomes instruments. Plast Reconstr Surg. 2007;120(4):823-37; discussion 838-9.

32. Rietjens M, Urban CA, Rey PC, Mazzarol G, Maisonneuve P, Garusi C, et al. Long-term oncological results of breast conservative treatment with oncoplastic surgery. Breast. 2007;16(4):387-95.

33. Spear SL, Boehmler JH, Taylor NS, Prada C. The role of the latissimus dorsi flap in reconstruction of the irradiated breast. Plast Reconstr Surg. 2007;119(1):1-9; discussion 10-1.
34. De Lorenzi F, Manconi A, Rietjens M, Petit JY. In response to: Rubino C, Dessy LA, Posadinu A. A modified technique for nipple reconstruction: the "arrow flap". Br J Plast Surg 2003;56:247. J Plast Reconstr Aesthet Surg. 2007;60(8):971-2.

35. Disa JJ, McCarthy CM, Mehrara BJ, Pusic AL, Cordeiro PG. Immediate latissimus dorsi/prosthetic breast reconstruction following salvage mastectomy after failed lumpectomy/irradiation. Plast Reconstr Surg. 2008;121(4):159e-64e.

36. Chen CM, Disa JJ, Sacchini V, Pusic AL, Mehrara BJ, GarciaEtienne CA, et al. Nipple-sparing mastectomy and immediate tissue expander/implant breast reconstruction. Plast Reconstr Surg. 2009;124(6):1772-80.

37. Zucca-Matthes G, Vieira RA. The value of patients' expectation on breast oncoplastic surgery. Breast J. 2014;20(6):676-8.

38. Haloua MH, Krekel NM, Winters HA, Rietveld DH, Meijer S, Bloemers FW, et al. A systematic review of oncoplastic breastconserving surgery: current weaknesses and future prospects. Ann Surg. 2013;257(4):609-20.

39. Cordeiro PG, PusicAL, Disa JJ, McCormick B, VanZeeK. Irradiation after immediate tissue expander/implant breast reconstruction: outcomes, complications, aesthetic results, and satisfaction among 156 patients. Plast Reconstr Surg. 2004;113(3):877-81.

40. Santos G, Urban C, Edelweiss MI, Zucca-Matthes G, de Oliveira VM, Arana GH, et al. Long-term comparison of aesthetical outcomes after oncoplastic surgery and lumpectomy in breast cancer patients. Ann Surg Oncol. 2015;22(8):2500-8.

41. Spear SL, Prada CA. Reduction mammaplasty in conjunction with breast conservation. Semin Plast Surg. 2004;18(3):255-60.

42. Bayram Y, Kulahci Y, Irgil C, Calikapan M, Noyan N. Skinreducing subcutaneous mastectomy using a dermal barrier flap and immediate breast reconstruction with an implant: a new surgical design for reconstruction of early-stage breast cancer. Aesthetic Plast Surg. 2010;34(1):71-7.

43. Rietjens M, De Lorenzi F, Manconi A, Lanfranchi L, Teixera Brandao LA, Petit JY. 'Ilprova', a surgical film for breast sizers: a pilot study to evaluate its safety. J Plast Reconstr Aesthet Surg. 2008;61(11):1398-9.

44. Carvalho FM, Bacchi LM, Pincerato KM, Van de Rijn M, Bacchi CE. Geographic differences in the distribution of molecular subtypes of breast cancer in Brazil. BMC Womens Health. 2014;14:102.

45. Liedke PE, Finkelstein DM, Szymonifka J, Barrios CH, ChavarriGuerra Y, Bines J, et al. Outcomes of breast cancer in Brazil related to health care coverage: a retrospective cohort study. Cancer Epidemiol Biomarkers Prev. 2014;23(1):126-33.

46. Gonzaga CM, Freitas-Junior R, Curado MP, Sousa AL, Souza-Neto JA, Souza MR. Temporal trends in female breast cancer mortality in Brazil and correlations with social inequalities: ecological timeseries study. BMC Public Health. 2015;15:96.

47. Vieira RAC, Matthes AGZ, Michelli RAD, Ribeiro GFP, Mendonça MLH, Bailáo-Jr A, et al. A oncoplastia e o tempo de treinamento do cirurgiấo. Rev Bras Mastologia. 2010;20(2):66-70.

48. Urban CA. New classification for oncoplastic procedures in surgical practice. Breast. 2008;17(4):321-2.

49. Cardoso MJ, Macmillan RD, Merck B, Munhoz AM, Rainsbury R. Training in oncoplastic surgery: an international consensus. The 7th Portuguese Senology Congress, Vilamoura, 2009. Breast. 2010;19(6):538-40. 
50. Rietjens M, Villa G, Toesca A, Rizzo S, Raimondi S, Rossetto F, et al. Appropriate use of magnetic resonance imaging and ultrasound to detect early silicone gel breast implant rupture in postmastectomy reconstruction. Plast Reconstr Surg. 2014;134(1):13e-20e.

51. Rezai M, Kellersmann S, Knispel S, Lax H, Kimmig R, Kern P. Translating the concept of intrinsic subtypes into an oncoplastic cohort of more than 1000 patients: predictors of recurrence and survival. Breast. 2015;24(4):384-90.

52. Loblaw DA, Prestrud AA, Somerfield MR, Oliver TK, Brouwers MC, Nam RK, et al. American Society of Clinical Oncology Clinical Practice Guidelines: formal systematic review-based consensus methodology. J Clin Oncol 2012;30(25):3136-40.

53. Hsu J, Brozek JL, Terraciano L, Kreis J, Compalati E, Stein AT, et al. Application of GRADE: making evidence-based recommendations about diagnostic tests in clinical practice guidelines. Implement Sci. 2011;6:62.
54. Coates AS, Winer EP, Goldhirsch A, Gelber RD, Gnant M, PiccartGebhart M, et al. Tailoring therapies improving the management of early breast cancer: St Gallen International Expert Consensus on the Primary Therapy of Early Breast Cancer 2015. Ann Oncol. 2015;26(8):1533-46.

55. Bertolini F, Petit JY, Kolonin MG. Stem cells from adipose tissue and breast cancer: hype, risks and hope. Br J Cancer. 2015;112(3):419-23.

56. Vallejo A, Urban C, Zucca-Matthes G, Rietjens M. Is there enough evidence to use lipofilling in breast cancer reconstruction? Plast Reconstr Surg. 2013;132(4):689e-691e.

57. Petit JY, Rietjens M, Botteri E, Rotmensz N, Bertolini F, Curigliano G, et al. Evaluation of fat grafting safety in patients with intraepithelial neoplasia: a matched-cohort study. Ann Oncol. 2013;24(6):1479-84

58. Petit JY, Botteri E, Lohsiriwat V, Rietjens M, De Lorenzi F, Garusi $\mathrm{C}$, et al. Locoregional recurrence risk after lipofilling in breast cancer patients. Ann Oncol. 2012;23(3):582-8. 